\title{
Quantum computation with ions in thermal motion
}

\author{
Anders Sørensen and Klaus Mølmer \\ Institute of Physics and Astronomy, University of Aarhus \\ DK-8000 Århus C
}

We propose an implementation of quantum logic gates via virtual vibrational excitations in an ion trap quantum computer. Transition paths involving unpopulated, vibrational states interfere destructively to eliminate the dependence of rates and revolution frequencies on vibrational quantum numbers. As a consequence quantum computation becomes feasible with ions whos vibrations are strongly coupled to a thermal reservoir.

Pacs. 03.67.Lx, 03.65 Bz, 89.70+c

Recently, methods to entangle states of two or several quantum systems in controlled ways have become subject of intense studies. Such methods may find applications in fundamental tests of quantum physics [1] and in precision spectroscopy [2]; and they offer fundamentally new possibilities in quantum communication and computing [3]. A major obstacle to these efforts is decoherence of the relevant quantum states. In many proposed implementations of quantum computation the quantum bits (qubits) are stored in physical degrees of freedom with long coherence times, like nuclear spins, and decoherence is primarily due to the environment interacting with the channel used to perform logic gates between qubits [ 4 ]. In this Letter we present a scheme which is insensitive to the interaction between the quantum channel and the environment. Specifically, we consider an implementation of quantum computation in an ion trap, but we hope to stimulate similar ideas to reduce decoherence in other physical implementations.

The ion trap was originally proposed by Cirac and Zoller [5] as a system with good experimental (optical) access and control of the quantum degrees of freedom, and they suggested an implementation of the necessary ingredients in terms of one-bit and two-bit operations to carry out quantum computation. In the ion trap computer, qubits are represented by internal states of the ions. The number of qubits equals the number of ions, and this system is scalable to the problem size in contrast to NMR quantum computation which is only applicable with a limited number of qubits [6].

The ion trap method [5] uses collective spatial vibrations for communication between ions, and it requires that the system is restricted to the joint motional ground state of the ions. For two ions this has recently been accomplished [7]. We present an alternative implementation of quantum gates that is both insensitive to the vibrational state and robust against changes in the vi- brational motion occurring during operation, as long as the ions are in the Lamb-Dicke regime, i.e., their spatial excursions are restricted to a small fraction of the wavelength of the exciting radiation. Our mechanism relies on features of quantum mechanics that are often responsible for "paradoxical effects": i) The vibrational degrees of freedom used for communication in our scheme only enter virtually i.e., although they are crucial as intermediate states in our processes, we never transfer population to states with different vibrational excitation. ii) Transition paths involving different unpopulated, vibrational states interfere destructively to eliminate the dependence of rates and revolution frequencies on vibrational quantum numbers.

Like in the original ion trap scheme [5], we address each ion with a single laser, but quantum logic gates involving two ions are performed through off-resonant laser pulses. For the laser addressing the first ion, we choose a detuning close to the upper sideband, i.e., close to resonance with a joint vibrational and internal excitation of the ion. We choose the detuning of the laser addressing the second ion to be the negative of the detuning of the first laser, see Fig. 11 (a). This laser setting couples the states $|g g n\rangle \leftrightarrow\{|e g n+1\rangle,|g e n-1\rangle\} \leftrightarrow|e e n\rangle$, where the first (second) letter denotes the internal state $e$ or $g$ of the first (second) ion and $n$ is the quantum number for the relevant vibrational mode of the trap. We choose the detuning from the sideband so large that the intermediate states $|e g n+1\rangle$ and $|g e n-1\rangle$ are not populated in the process. As we shall show below, the internal state transition is insensitive to the vibrational quantum number $n$, and it may be applied even with ions which exchange vibrational energy with a surrounding reservoir.

If we tune the lasers sufficiently close to the sidebands we can neglect all other vibrational modes and concentrate on one collective degree of vibrational excitation of the ions [8]. In this case our system can be described by the following Hamiltonian

$$
\begin{aligned}
H & =H_{0}+H_{\mathrm{int}} \\
H_{0} & =\hbar \nu\left(a^{\dagger} a+1 / 2\right)+\hbar \omega_{e g} \sum_{i} \sigma_{z i} / 2 \\
H_{\mathrm{int}} & =\sum_{i} \frac{\hbar \Omega_{i}}{2}\left(\sigma_{+i} e^{i\left(\eta_{i}\left(a+a^{\dagger}\right)-\omega_{i} t\right)}+\text { h.c. }\right),
\end{aligned}
$$

where $\nu$ is the frequency and $a^{\dagger}$ and $a$ are the ladder operators of the vibrational mode and $\hbar w_{e g}$ is the energy difference between the internal states $e$ and $g$. Pauli matrices $\sigma_{i}$ represent the internal degrees of freedom for the 
$i$ 'th ion, and $\omega_{i}$ and $\Omega_{i}$ are the frequency and Rabi frequency of the laser addressing the $i$ 'th ion. [ In a practical realization, one might use Raman transitions between low lying states of the ions due to their long coherence time. By appropriate redefinition of the symbols our formalism also describes this implementation [9].] We consider an ion trap operating in the Lamb-Dicke limit, i.e. the ions are cooled to a regime with vibrational quantum numbers $n$ ensuring that $\eta_{i} \sqrt{n+1}$ is well below unity (Note that this may still allow n-values well above unity.) In our analytical calculations we use an expansion of $H_{\text {int }}$ to second order in $\eta_{i}$, but in our numerical treatment we apply the exact Hamiltonian (1).

We wish to perform an operation on the mutual state of two ions 1 and 2 selected freely within the string of ions, and we assume that $\eta_{1}=\eta_{2}=\eta$ and $\Omega_{1}=\Omega_{2}=\Omega$. With the choice of detunings described above, the only energy conserving transitions are between $|g g n\rangle$ and $|e e n\rangle$. The Rabi frequency $\tilde{\Omega}$ for the transition between these states, via intermediate states $m$, can be determined in second order perturbation theory,

$$
\left(\frac{\tilde{\Omega}}{2}\right)^{2}=\frac{1}{\hbar^{2}}\left|\sum_{m} \frac{\left\langle e e n\left|H_{\mathrm{int}}\right| m\right\rangle\left\langle m\left|H_{\mathrm{int}}\right| g g n\right\rangle}{E_{g g n}+\hbar \omega_{i}-E_{m}}\right|^{2},
$$

where the laser energy $\hbar \omega_{i}$ is the energy of the laser addressing the ion which is excited in the intermediate state $|m\rangle$. If we restrict the sum to $|e g n+1\rangle$ and $|g e n-1\rangle$, we get

$$
\tilde{\Omega}=-\frac{(\Omega \eta)^{2}}{2(\nu-\delta)}
$$

where $\delta=\omega_{1}-\omega_{e g}$ is the detuning of the laser addressing the first ion [10].

The remarkable feature in Eq. (3) is that it contains no dependence on the vibrational quantum number $n$. This is due to interference between the two paths indicated in Fig. 11(b). If we take the path where ion No. 1 is excited first, we have a factor of $n+1$ appearing in the enumerator $(\sqrt{n+1}$ from raising and $\sqrt{n+1}$ from lowering the vibrational quantum number). In the other path we obtain a factor of $n$. Due to the opposite detunings, the denominators in Eq. (2) have opposite signs and the $n$ dependence disappears when the two terms are subtracted. The coherent evolution of the internal atomic state is thus insensitive to the vibrational quantum numbers, and it may be observed with ions in any superposition or mixture of vibrational states.

From the above arguments we expect to see perfect sinusoidal oscillations between the population of the internal states $|g g\rangle$ and $|e e\rangle$. To confirm the validity of our pertubative analysis we have performed a direct numerical integration of the Scrödinger equation with the Hamiltonian (11) to all orders in $\eta$. We have considered a situation, where both ions are initially in the internal ground state. For the vibrational state, we have investigated a number of different states, including Fock, coherent and thermal states, all yielding qualitatively similar results. The outcome of the computation for a coherent state of vibrational motion can be seen in Fig. 2, where we show the evolution of relevant terms of the atomic internal state density matrix $\rho_{i j, k l}=T r_{n}(\rho|k l\rangle\langle i j|)$, where $i, j, k, l=e$ or $g$, and where $T r_{n}$ denotes the partial trace over the unobserved vibrational degrees of freedom. The figure clearly shows that we have Rabi oscillations between the atomic states $|g g\rangle$ and $|e e\rangle$, and the values of the off diagonal element $\rho_{g g, e e}$ confirm that we have a coherent evolution of the internal atomic state which is not entangled with the vibrational motion. Superimposed on the sinusoidal curves are small oscillations with a high frequency due to off resonant couplings of the type $|g g n\rangle \rightarrow|e g n+1\rangle,|g g n\rangle \rightarrow|g e n-1\rangle$ and $|g g n\rangle \rightarrow|e g n\rangle$. The magnitude of these oscillation and the deviation from ideal transfer between $|g g\rangle$ and $|e e\rangle$ can be suppressed by decreasing $\Omega$.

The analysis given so far is sufficient for creation of internal state entanglement, completely decoupled from the external motion of the ions. By optical pumping we can prepare a state $\rho=|g g\rangle\langle g g| \otimes \rho_{v i b}$, and if we apply radiation fields corresponding to a pulse of duration $T=$ $\frac{\pi}{2|\hat{\Omega}|}$, our system is described by the density operator $\rho=$ $|\psi\rangle\langle\psi| \otimes \rho_{v i b}$, where $|\psi\rangle$ is a maximally entangled EPRstate $\frac{1}{\sqrt{2}}(|g g\rangle-i|e e\rangle)$.

Since the states $|e g\rangle$ and $|g e\rangle$ do not fulfill any resonance condition one might expect that they are unaffected by the laser pulses. Due to $n$-dependent perturbations of the energy levels by the lasers this is, however, not the case. Keeping only the most important terms, we get the energy shifts

$$
\begin{aligned}
& \Delta E_{g g n}=\Delta E_{e e n}=-\hbar \frac{(\eta \Omega)^{2}}{4} \frac{1}{\nu-\delta} \\
& \Delta E_{\text {egn }}=\hbar\left(\frac{(\eta \Omega)^{2}}{2} \frac{n}{\nu-\delta}-\frac{\Omega^{2}}{2 \delta}\right) \\
& \Delta E_{g e n}=\hbar\left(-\frac{(\eta \Omega)^{2}}{2} \frac{n+1}{\nu-\delta}+\frac{\Omega^{2}}{2 \delta}\right) .
\end{aligned}
$$

The energy shifts of the $|e e n\rangle$ and $|g g n\rangle$ are identical and independent of $n$, but since the energy shifts of $|e g n\rangle$ and $|g e n\rangle$, depend on the vibrational quantum number, the time evolution introduces phase factors $e^{-i \Delta E_{e g n} t / \hbar}$, which depend on $n$, and e.g., at the time $t=T_{i n v}=$ $\frac{2 \pi(\nu-\delta)}{\eta^{2} \Omega^{2}}$ where $|g g\rangle$ end $|e e\rangle$ are inverted, factors of $(-1)^{n}$ will tend to extinguish the coherence between internal states $|e e\rangle$ and $|e g\rangle$. This coherence can be restored by a trick resembling photon echoes [11]. Notice that the $n$ dependent part of $\Delta E_{\text {egn }}$ is minus the $n$ dependent part of $\Delta E_{g e n}$. If at any time $T / 2$ we change the sign of the laser detuning $\delta$, phase components proportional to $n$ will begin to rotate in the opposite direction and at 
time $T$ we will have a revival of the coherence. This is confirmed by our numerical solution of the Schrödinger equation presented in Fig. 3, where we change the laser detunings at the time $T_{i n v} / 2$ and at the time $T_{i n v}$ we have completed the transfer $\frac{1}{\sqrt{2}}(|g g\rangle+|e g\rangle) \rightarrow \frac{1}{\sqrt{2}}(-i|e e\rangle+$ $|e g\rangle)$.

No particularly demanding assumptions have been made for the experimental parameters. With a vibrational frequency $\nu / 2 \pi=200 \mathrm{kHz}$, the transition shown in Fig. 2, require Rabi frequencies $\Omega / 2 \pi$ of modest 20 $\mathrm{kHz}$, and the evolution from $|g g\rangle$ to $|e e\rangle$ is accomplished in $5 \mathrm{~ms}$. To be relevant for real computational tasks it is necessary that our evolution is robust against decoherence effects on this long time scale. An important source of decoherence is heating of the vibrational motion, and it is a major asset of our proposal that it can be made insensitive to the interaction with the environment: The arguments leading to Eq. (3) do not require that the ions remain in the same vibrational state, and the coherent oscillation from $|g g\rangle$ to $|e e\rangle$ may still be observed when the vibrational motion exchange energy with a thermal reservoir. The photon echo trick, however, is sensitive to heating: If the vibrational quantum number $n$ change its value at the time $T / 2$ where the detunings are inverted, the second half of the gate, will no longer revert the phase evolution due to the new value of $n$ and coherence is lost. If instead the detunings are inverted $N$ times during a gate, the erroneous phase will only be on the order of the phase evolution in time $T / N$, and the effect of the heating is reduced.

Rather than inverting the detunings a large number of times, we suggest to continuously apply lasers with both detunings $\pm \delta$ on both ions. With two fields of opposite detunings and identical Rabi frequency $\Omega$ there are two contributing paths in addition to the two paths in Fig. 1. The contribution from the two additional paths are identical to the two original paths and the only modifications to the $|g g\rangle \leftrightarrow|e e\rangle$ Rabi frequency in Eq. (3) is multiplication by a factor of two. With bichromatic fields there also exists a resonant transition from $|e g\rangle$ to $|g e\rangle$. The Rabi frequency of this transition is the negative of the Rabi frequency from $|g g\rangle$ to $|e e\rangle$ and the evolution will be described by

$$
\begin{aligned}
& |g g\rangle \rightarrow \cos \left(\frac{\tilde{\Omega} T}{2}\right)|g g\rangle+i \sin \left(\frac{\tilde{\Omega} T}{2}\right)|e e\rangle \\
& |e e\rangle \rightarrow \cos \left(\frac{\tilde{\Omega} T}{2}\right)|e e\rangle+i \sin \left(\frac{\tilde{\Omega} T}{2}\right)|g g\rangle \\
& |g e\rangle \rightarrow \cos \left(\frac{\tilde{\Omega} T}{2}\right)|g e\rangle-i \sin \left(\frac{\tilde{\Omega} T}{2}\right)|e g\rangle \\
& |e g\rangle \rightarrow \cos \left(\frac{\tilde{\Omega} T}{2}\right)|e g\rangle-i \sin \left(\frac{\tilde{\Omega} T}{2}\right)|g e\rangle .
\end{aligned}
$$

To validate that the evolution in Eq. (5) is in fact stable against heating we introduce a thermal reservoir described by relaxation operators $c_{1}=\sqrt{\Gamma\left(1+n_{\text {therm }}\right)} a$ and $c_{2}=\sqrt{\Gamma n_{\text {therm }}} a^{\dagger}$, where $\Gamma$ characterizes the strength of the interaction and $n_{\text {therm }}$ is the mean vibrational number in thermal equilibrium. We analyse the dynamics of the system using Monte Carlo wavefunctions [12], which evolve with a non Hermitian Hamiltonian interrupted by jumps at random times. The result of the computation can be seen in Fig. 4, where we show (left) the result of a single Monte Carlo realization with quantum jumps indicated by arrows and (right) the average over 10 realizations. In the figure we have chosen $n_{\text {therm }}=2$. This rather low value could represent a heating mechanism counteracted by laser cooling on a particular ion reserved for this purpose. In the simulations 34 vibrational quanta are exchanged with the reservoir on average, and we wish to emphasize that with the proposed scheme the gate is almost unaffected even though the duration of the gate is much longer than the coherence time of the channel used to communicate between the qubits.

It has been proven that any unitary evolution involving a number of qubits can be constructed using only single qubit operations and a simple universal quantum gate like for instance the two-qubit control-not operation [13]. With the evolution described by Eq. (5) a control-not operation is created by the following sequence of operations: $P_{1}, P_{2}^{-1}, H_{2}, R, P_{1}, H_{1}, P_{1}, R$ and $P_{2}$, where $R$ is the evolution in Eq. (5) with $T=\frac{\pi}{2|\tilde{\Omega}|}, P_{i}$ is a $\pi / 2$ phase change of $|e\rangle$ in ion $i$, and $H_{i}$ is a Hadamard transformation on ion $i$. With ready access to one-qubit operations in the ion trap we thus have available the ingredients for successful quantum computation.

We note that with only two ions in the trap, the use of bichromatic light leads to the evolution (5) even without individual optical access, and if many ions are illuminated by the same field, multi particle entanglement is created [14]. As a further remarkable feature of our implementation, we note how easy it is to simultaneously operate on different pairs of ions: If we wish to apply our gate on the pairs $(i, j)$ and $(k, l)$, we simply illuminate ions $i$ and $j$ with fields of detunings $\pm \delta_{i j}$ and ions $k$ and $l$ with another pair of detunings $\pm \delta_{k l}$. The only resonant transitions are the ones of the two desired gates and, however mind boggling this may be, the virtually excited but never populated vibrational mode has been used for two processes at the same time.

Note added: Since submission of this work, two proposals for computing with vibrationally excited ions have appeared. 15 uses widely separated vibrational states, and can hardly be generalized beyond 2 or 3 qubits; 16] involves dynamics which is conditioned on the vibrational state and is not applicable during heating. 
[1] D. M. Greenberger, M. A. Horne and A. Zeilinger, Physics Today 46, 22-29, August 1993.

[2] J. J. Bollinger, W. M. Itano, D. J. Wineland and D. J. Heinzen, Phys. Rev. A 54, 4649 (1996).

[3] Physics World 11, 33-57, March 1998. Special issue on Quantum Information.

[4] B. E. Kane, Nature 393, 133 (1998), T. Pellizari, S. A. Gardiner, J. I. Cirac and P. Zoller, Phys. Rev. Lett. 75, 3788 (1995).

[5] J. I. Cirac and P. Zoller, Phys. Rev. Lett. 74, 4091 (1995).

[6] I. L. Chuang, L. M. K. Vandersypen, X. Zhou, D. W. Leung and S. Lloyd, Nature vol. 393, 143 (1998); I. L. Chuang, N. Gershenfeld and M. Kubinec, Phys. Rev. lett. 80, 3408 (1998); J. Jones, M. Mosca and R. H. Hansen, Nature vol 393, 344 (1998).

[7] B. E. King, C. S. Wood, C. J. Myatt, Q. A. Turchette, D. Leibfried, W. M. Itano, C. Monroe, D. J. Wineland, Phys. Rev. Lett. 81, 1525 (1998); Q. A. Turchette, C. S. Wood, B. E. King, C. J. Myatt, D. Liebfried, W. M. Itano, C. Monroe and D. J. Wineland, Phys. Rev. Lett. 81, 3631 (1998).

[8] Numerical calculations retaining two collective vibrations for a two ion system have confirmed the validity of the omission of the far off-resonant vibration modes.

[9] D. M. Meekhof, C. Monroe, B. E. King, W. M. Itano and D. J. Wineland, Phys. Rev. Lett. 76, 1796 (1996).

[10] If the additional coupling to the lower (higher) sideband of ion $1(2)$ is included $2(\nu-\delta)$ in Eq. (3) is replaced by $\left(\nu^{2}-\delta^{2}\right) / \nu$

[11] L. Allan and J. M. Eberly, 'Optical resonance and twolevel atoms' Ch. 9, Dover, New York, 1987.

[12] K. Mølmer, Y. Castin and J. Dalibard, J. Opt. Soc. Am. B 10524 (1993).

[13] A. Barenco, C. H. Bennet, R. Cleve, D. P. Divincenzo, N. Margolus, P. Shor, T. Sleator, J. A. Smolin and H. Weinfurter, Phys. Rev. A 55, 3457 (1995).

[14] K. Mølmer and A. Sørensen, Schrödinger's cat in a hot trap, quant-ph 9810040 .

[15] J. F. Poyatos, J. I. Cirac and P. Zoller, Phys. Rev. Lett. 81, 1322 (1998).

[16] S. Schneider, D. F. V. James and G. J. Milburn, quant-ph 9808012 . (a)

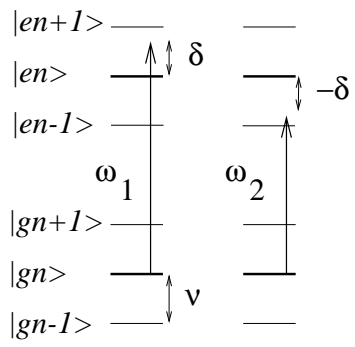

(b)

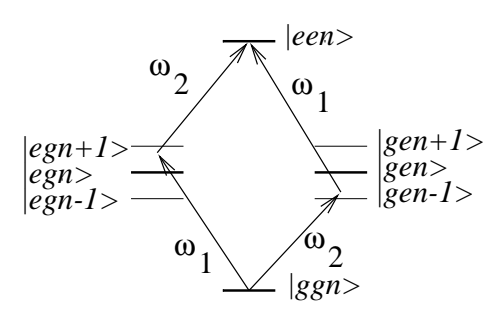

FIG. 1. Energy levels and laser detunings. (a) Two ions with quantised vibrational motion are illuminated with lasers detuned close to the upper and lower sidebands. (b) The ions oscillate in collective vibrational modes, and two interfering transition paths are identified.

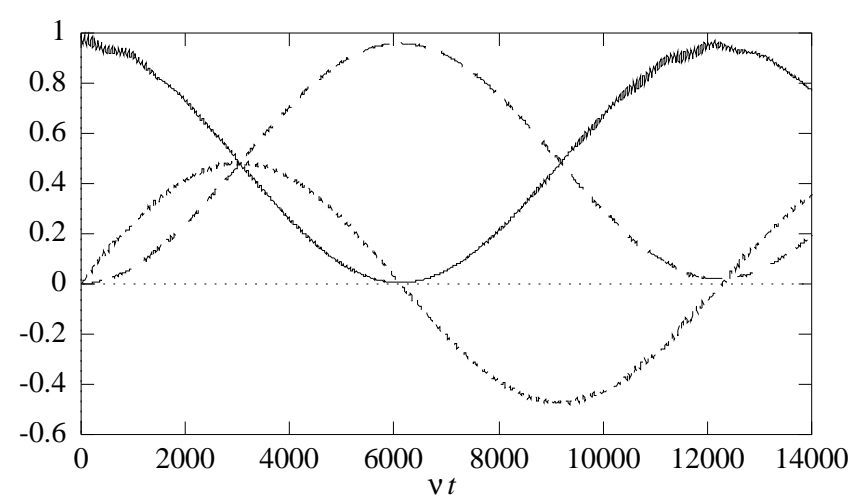

FIG. 2. Rabi oscillations between $|g g\rangle$ and $|e e\rangle$. The figure shows the time evolution of the internal atomic density matrix elements $\rho_{g g, g g}$ (full line), $\rho_{e e, e e}$ (long dashed line) and $\operatorname{Im}\left(\rho_{g g, e e}\right)$ (short dashed line). The magnitude of $\operatorname{Re}\left(\rho_{g g, e e}\right)$ is below 0.03 and is not shown. In the initial state, the ions are in the internal ground state and a coherent vibrational state with mean excitation $\bar{n}=2$. Parameters are $\delta=0.90 \nu$, $\Omega=0.10 \nu$ and $\eta=0.10$

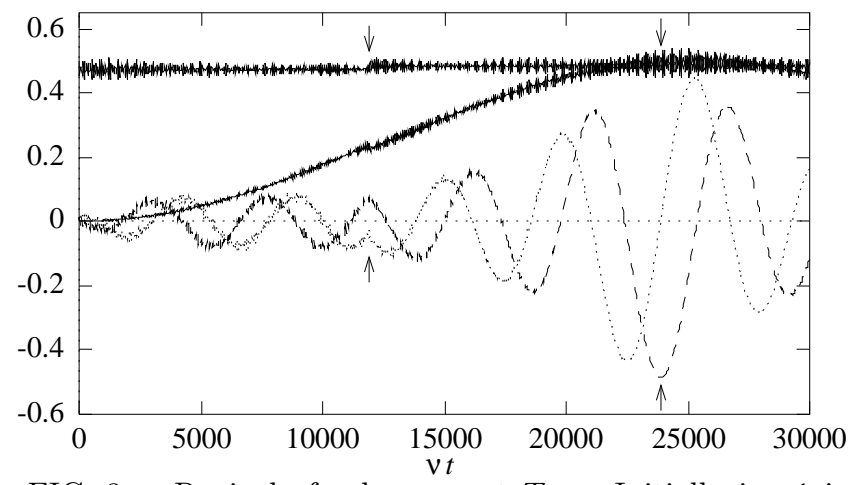

FIG. 3. Revival of coherence at $T_{i n v}$. Initially ion 1 is in a superposition $\frac{1}{\sqrt{2}}(|g\rangle+|e\rangle)$ and ion 2 is in $|g\rangle$. The sign of $\delta$ is changed at $T_{i n v} / 2$ (left arrows), to ensure the perfect transition at $T_{i n v}$ (right arrows). The full lines represent populations of $|e e\rangle$ and $|e g\rangle$. The dotted and the dashed curves represent the real and imaginary part of the coherence $\rho_{e e, e g}$. Parameters are $\delta=0.90 \nu, \Omega=0.05 \nu$ and $\eta=0.1$
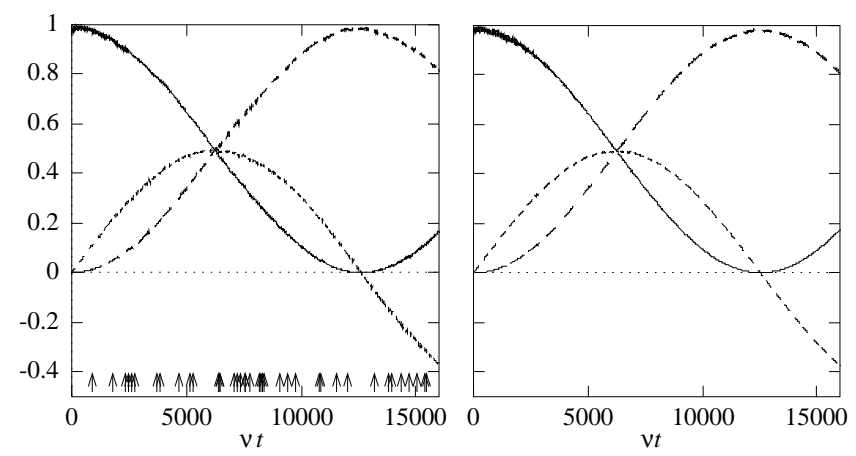
FIG. 4. Rabi oscillations in a heating trap. The left panel shows the result of a single Monte Carlo realization with a total of 39 jumps occuring at times indicated by the arrows. The right panel is an average over 10 realizations. The curves represent $\rho_{g g, g g}$ (full line), $\rho_{e e, e e}$ (long dashed) and $\operatorname{Im}\left(\rho_{g g, e e}\right)$ (short dashed). Parameters are $\delta=0.90 \nu, \Omega=0.10 \nu$, $\eta=0.1, \Gamma=2 * 10^{-4} \nu$ and $n_{\text {term }}=2$. 